\title{
Lactate Dehydrogenase as a Biomarker for Prediction of Refractory Mycoplasma pneumoniae Pneumonia in Children
}

\author{
Aizhen Lu MD PhD, Chuankai Wang, Xiaobo Zhang MD PhD, Libo Wang MD, and \\ Liling Qian MD PhD
}

\begin{abstract}
BACKGROUND: Corticosteroids have been used for refractory Mycoplasma pneumoniae pneumonia and have beneficial effects. The aim of this study was to identify the biomarkers for predicting refractory $M$. pneumoniae pneumonia in a timely fashion to initiate steroid therapy. METHODS: This was a prospective cohort study of children with $M$. pneumoniae pneumonia admitted to the Children's Hospital of Fudan University from September 2012 to August 2013. Lactate dehydrogenase (LDH) and other laboratory tests, including complete blood counts, C-reactive protein, erythrocyte sedimentation rate (ESR), alanine aminotransferase, aspartate aminotransferase, $\alpha$-hydroxybutyrate dehydrogenase (HBDH), creatine kinase, and creatine kinase MB, were performed on admission. Based on the definition of refractory M. pneumoniae pneumonia, subjects were divided into 2 groups: refractory $M$. pneumoniae pneumonia and usual M. pneumoniae pneumonia. The diagnostic values of laboratory findings were analyzed. RESULTS: In total, 653 subjects were enrolled, including 300 in the refractory pneumonia group and 353 in the usual pneumonia group. There was no significant difference in sex distribution between the 2 groups. The average age in the refractory M. pneumoniae pneumonia group was greater than that in the usual M. pneumoniae pneumonia group. Compared with the usual pneumonia group, the refractory pneumonia group showed significantly higher levels of C-reactive protein, serum LDH, serum HBDH, serum alanine aminotransferase, serum aspartate aminotransferase, and neutrophils and higher ESRs. Logistic regression showed that age, LDH, and ESR were the significant factors in predicting refractory M. pneumoniae pneumonia. In addition, LDH and HBDH were strongly correlated, and receiver operating characteristic curve analysis showed that the area under the curve of LDH was 0.718 with a cutoff of $379 \mathrm{IU} / \mathrm{L}$, that of ESR was 0.683 with a cutoff of $32.5 \mathrm{IU} / \mathrm{L}$, and that of $\mathrm{HBDH}$ was 0.691 with a cutoff of $259.5 \mathrm{IU} / \mathrm{L}$. CONCLUSIONS: Serum LDH can be used as a biomarker to predict refractory M. pneumoniae pneumonia at the early stage of hospitalization. Key words: Refractory Mycoplasma pneumoniae pneumonia; lactate dehydrogenase; $\alpha$-hydroxybutyrate dehydrogenase; erythrocyte sedimentation rate; receiver operating curve. [Respir Care 2015;60(10):1469-1475. (C) 2015 Daedalus Enterprises]
\end{abstract}

\section{Introduction}

Mycoplasma pneumoniae is one of the major causative pathogens of community-acquired respiratory tract infec-

The authors are affiliated with the Respiratory Department, Children's Hospital of Fudan University, Shanghai, China.

This study was supported by the Program for New Century Excellent Talents in University (NCET-12-0126). The authors have disclosed no conflicts of interest.

Correspondence: Libo Wang MD and Liling Qian MD PhD, Children's tions in children. M. pneumoniae infections occur both endemically and in cyclic epidemics every 3-7 y and account for $10-40 \%$ of community-acquired pneumonia cases in children. ${ }^{1}$ Although pneumonia due to M. pneumoniae is usually a benign, self-limited disease, some cases become refractory or severe and life-threatening. ${ }^{2-4}$ It can also cause a wide array of extrapulmonary manifestations ${ }^{5-9}$ and even lead to sequelae and disability, such as bronchi-

Hospital of Fudan University, 399 Wan Yuan Road, Shanghai 201102, China. E-mail: wanglbc@163.com and 1lqian@126.com. 


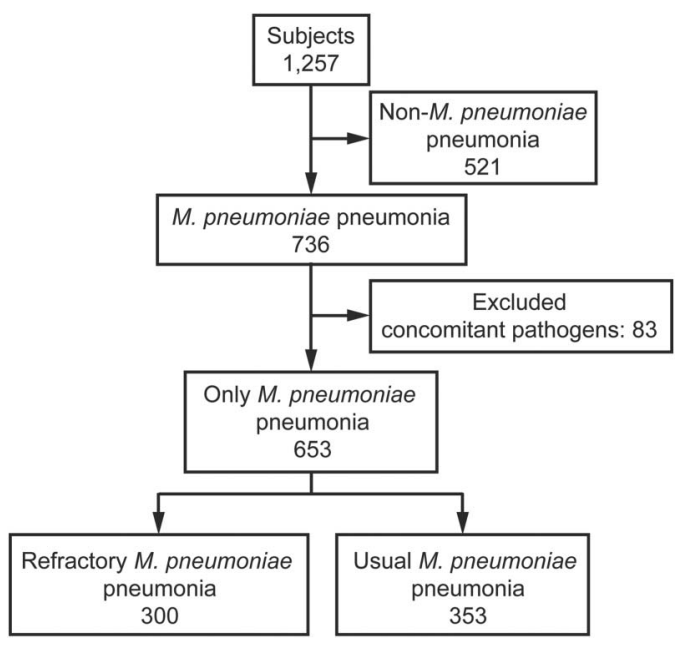

Fig. 1. Flow chart.

ectasis, bronchiolitis obliterans, interstitial pulmonary fibrosis, and paralysis. ${ }^{10,11}$

Clinically, corticosteroids have been used for refractory M. pneumoniae pneumonia and have a dramatic beneficial effect in both children and adults. ${ }^{2,4,12}$ Clinicians must be cognizant of children with $M$. pneumoniae pneumonia at risk for developing refractory or serious illnesses and initiate steroid therapy in a timely fashion. Thus, it is desirable to predict refractory illness as soon as possible.

In a previous study, ${ }^{4}$ we found that lactate dehydrogenase (LDH), C-reactive protein, and erythrocyte sedimentation rate (ESR) on admission were increased in subjects with refractory M. pneumoniae pneumonia. We hypothesized that they play important roles in this illness. Based on a review of the literature, there are a few reports on the role of LDH, C-reactive protein, and ESR in refractory M. pneumoniae pneumonia, but they are limited to case series. ${ }^{4,13,14}$ In this study, we explored whether LDH, Creactive protein, and ESR are potential laboratory markers that could be used to predict refractory M. pneumoniae pneumonia in a large sample.

\section{Methods}

\section{Subjects and Groups}

This was a planned secondary analysis of a prospective cohort study (Fig. 1). Children with M. pneumoniae pneumonia admitted to the Children's Hospital of Fudan University from September 2012 to September 2013 were

DOI: $10.4187 /$ respcare. 03920

\section{QUICK LOOK}

\section{Current knowledge}

M. pneumoniae is one of the major causative pathogens of community-acquired respiratory tract infections in children. Although pneumonia due to M. pneumoniae is usually benign, some cases develop into refractory, lifethreatening disease. Corticosteroids are an effective treatment for severe disease. A biomarker or method to predict patients at risk for developing refractory disease could improve the timing of corticosteroid treatment and improve patient outcomes.

\section{What this paper contributes to our knowledge}

In a group of children admitted to the hospital for M. pneumoniae pneumonia, serum lactate dehydrogenase (LDH) predicted refractory disease at hospitalization. LDH may prove useful as a biomarker for predicting refractory disease and determining which candidates may benefit from early corticosteroid therapy.

enrolled. M. pneumoniae infection was confirmed by serologic testing to detect $M$. pneumoniae immunoglobulin M (IgM) by enzyme-linked immunosorbent assay and/or polymerase chain reaction testing for M. pneumoniae in nasopharyngeal secretions. Subjects with immune deficiencies, chronic diseases, or heart diseases or who were using immunosuppressive drugs were excluded. All enrolled subjects had negative tuberculosis IgM or purified protein derivative tests. In addition, their nasopharyngeal secretions were negative for respiratory syncytial viruses, influenza viruses, adenovirus, parainfluenza virus, and Chlamydia trachomatis. The subjects also had negative bacterial cultures of nasopharyngeal secretions and double-negative blood cultures. Consent for participation was obtained. The study was approved by the hospital's ethics committee.

Refractory M. pneumoniae pneumonia is defined as a case with prolonged fever accompanied by deterioration of radiological findings despite appropriate management with macrolide treatment for $\geq 7 \mathrm{~d}^{2}{ }^{2,15,16}$ On the basis of this definition, we reviewed subjects' records and divided them into 2 groups: a refractory $M$. pneumoniae pneumonia group and a usual M. pneumoniae pneumonia group.

\section{Study Variables and Data Collection}

The main outcome measure of the study was serum LDH on admission. We also obtained complete blood counts, ESRs, and levels of C-reactive protein, alanine 
Lactate Dehydrogenase as a Biomarker for M. pneumoniae Pneumonia

Table 1. Comparison of Laboratory Findings on Admission for the Refractory and Usual M. pneumoniae Pneumonia Groups

\begin{tabular}{|c|c|c|c|}
\hline Laboratory Test & $\begin{array}{l}\text { Refractory } M . \text { pneumoniae } \\
\text { Pneumonia Group }\end{array}$ & $\begin{array}{l}\text { Usual } M . \text { pneumoniae } \\
\text { Pneumonia Group }\end{array}$ & $P$ \\
\hline Creatine kinase, IU/L & $116 \pm 1,266(13-986,74)$ & $89 \pm 70(2-825,72)$ & .85 \\
\hline Creatine kinase $\mathrm{MB}, \mathrm{IU} / \mathrm{L}$ & $35.7 \pm 35.7(9-189,24)$ & $30.2 \pm 21.0(6-230,25)$ & .40 \\
\hline LDH, IU/L & $449 \pm 258$ & $304 \pm 78.1$ & $<.01$ \\
\hline HBDH, IU/L & $357 \pm 233$ & $249 \pm 69.3$ & $<.01$ \\
\hline Alanine aminotransferase, IU/L & $23.2 \pm 42.8(1-394,11.5)$ & $12.4 \pm 18.2(1-219,8.5)$ & $<.01$ \\
\hline Aspartate aminotransferase, IU/L & $30.1 \pm 28.7(1-242,21)$ & $21.9 \pm 20.7(2-249,18)$ & $<.01$ \\
\hline White blood cells, $\times 10^{9} / \mu \mathrm{L}$ & $8.62 \pm 3.81(2.1-28.9,7.85)$ & $8.23 \pm 3.99(1.8-43.9,7.86)$ & $<.01$ \\
\hline Neutrophils, \% & $61.9 \pm 15.0(12.9-90.7,63.7)$ & $51.7 \pm 17.1(1.1-85.8,54.0)$ & $<.01$ \\
\hline Lymphocytes, \% & $28.8 \pm 13.5(3.6-75.7,27.0)$ & $39.3 \pm 16.3(3.40-91.2,35.6)$ & $<.01$ \\
\hline Platelets, $\times 10^{9} / \mu \mathrm{L}$ & $315 \pm 125(88-936,299)$ & $320 \pm 116(24-710,315)$ & .23 \\
\hline C-reactive protein, $\mathrm{mg} / \mathrm{L}$ & $31.4 \pm 39.1(7-161,7)$ & $15.5 \pm 20.5(7-161,7)$ & $<.01$ \\
\hline $\mathrm{ESR}, \mathrm{mm} / \mathrm{h}$ & $40.8 \pm 23.4(6-125,36)$ & $28.2 \pm 20.0(0-102,21)$ & $<.01$ \\
\hline \multicolumn{4}{|c|}{$\begin{array}{l}\text { Values are presented as mean } \pm \mathrm{SD} \text { (range, median). } \\
\mathrm{LDH}=\text { lactate dehydrogenase } \\
\mathrm{HBDH}=\alpha \text {-hydroxybutyrate dehydrogenase } \\
\mathrm{ESR}=\text { erythrocyte sedimentation rate }\end{array}$} \\
\hline
\end{tabular}

aminotransferase, aspartate aminotransferase, and other enzymes, including serum $\alpha$-hydroxybutyrate dehydrogenase (HBDH), creatine kinase, and creatine kinase MB. Demographic characteristics were collected from the subjects' records.

\section{Respiratory Pathogens}

Nasopharyngeal aspirates were tested for respiratory pathogens using a real-time, multiplex polymerase chain reaction assay (Diagnostic Hybrids, Athens, Ohio) in our hospital's clinical virology laboratory. The specific pathogens identified included influenza $\mathrm{A}$ and $\mathrm{B}$, respiratory syncytial viruses, adenovirus, parainfluenza virus, C. trachomatis, and $M$. pneumoniae. A positive polymerase chain reaction (Shanghai Shen Yousheng Biotech, Shanghai, China) result for $M$. pneumoniae was a copy number of $>2,500 / \mathrm{mL}$. Bacterial culture results based on nasopharyngeal aspirates and blood were obtained from the hospital's microbiology laboratory. M. pneumoniae IgM was detected using a commercial enzyme-linked immunosorbent assay kit (Beijing Rongzhi Haida Biotech, Beijing, China) according to the manufacturer's instructions, and the positive result was an M. pneumoniae IgM titer of $>1: 320$.

\section{Statistical Analysis}

Statistical analysis was performed with SPSS 16.0 (SPSS, Chicago, Illinois) and MedCalc (MedCalc Software, Mariakerke, Belgium), and $P<.05$ was considered statistically significant. Categorical variables were analyzed using the chi-square test. Normally distributed mea- surement data were analyzed with $t$ tests, and non-normally distributed measurement data were analyzed with Mann-Whitney $U$ tests. Multivariate analysis was performed using a stepwise logistic regression model. Receiver operating characteristic (ROC) curves were used to analyze the power of the laboratory markers for prediction of refractory $M$. pneumoniae pneumonia.

\section{Results}

\section{Demographical Characteristics}

We enrolled 653 subjects. There were 300 cases (171 boys and 129 girls) in the refractory $M$. pneumoniae pneumonia group, and 353 cases (221 boys and 132 girls) in the usual M. pneumoniae pneumonia group. There was no significant difference in sex distribution between the 2 groups $(P=.15)$. The average age in the refractory $M$. pneumoniae pneumonia group was $66.8 \pm 37.5$ months (range of 3-184 months, median of 66 months) and $51.4 \pm 34.4$ months (range of 1-156 months, median of 46 months) in the usual M. pneumoniae pneumonia group. The average age in the refractory pneumonia group was greater than that in the usual pneumonia group $(P<.001)$.

\section{Laboratory Findings}

The laboratory findings on admission are summarized in Table 1. Compared with the usual M. pneumoniae pneumonia group, the refractory $M$. pneumoniae pneumonia group showed significantly higher levels of C-reactive protein $(31.4 \pm 39.1$ vs $15.5 \pm 20.5 \mathrm{mg} / \mathrm{L}, P<.01)$, neutrophils $(61.9 \pm 15.0 \%$ vs $51.7 \pm 17.1 \%, P<.01)$, serum $\mathrm{LDH}$ 
Table 2. Logistic Regression Analysis of Associated Factors in Refractory M. pneumoniae Pneumonia

\begin{tabular}{lcccccc}
\hline \hline \multirow{2}{*}{ Relevant Factor } & B & SE & Wald & $P$ & Odds Ratio & \multicolumn{2}{c}{$95 \%$ CI for OR } \\
\cline { 4 - 7 } & & & & & Lower & Upper \\
Age & 0.014 & 0.004 & 10.197 & .001 & 1.01 & 1.00 \\
LDH & 0.009 & 0.001 & 33.821 & $<.001$ & 1.01 & 1.00 \\
ESR & 0.020 & 0.007 & 8.081 & .004 & 1.02 & 1.01 \\
\end{tabular}

$\overline{\mathrm{LDH}}=$ lactate dehydrogenase

$\mathrm{ESR}=$ erythrocyte sedimentation rate

$(449 \pm 258$ vs $304 \pm 78.1 \mathrm{IU} / \mathrm{L}, P<.01)$, serum $\mathrm{HBDH}$

$(357 \pm 233$ vs $249 \pm 69.3 \mathrm{IU} / \mathrm{L}, P<.001)$, serum alanine aminotransferase $(23.2 \pm 42.8 \mathrm{vs}$ $12.4 \pm 18.2 \mathrm{IU} / \mathrm{L}, P<.001)$, and serum aspartate aminotransferase $(30.1 \pm 28.7$ vs $21.9 \pm 20.7 \mathrm{IU} / \mathrm{L}, P<.01)$ and higher ESRs $(40.8 \pm 23.4$ vs $28.2 \pm 20.0 \mathrm{~mm} / \mathrm{h}, P<.01)$. However, the percentage of lymphocytes in the refractory M. pneumoniae pneumonia group was lower than that in the usual $M$. pneumoniae pneumonia group $(28.8 \pm 13.5 \%$ vs $39.3 \pm 16.3 \%, P<.01)$. The other laboratory findings were not significantly different between the 2 groups.

\section{Logistic Regression}

Age, LDH, HBDH, alanine aminotransferase, aspartate aminotransferase, neutrophils, lymphocytes, C-reactive protein, and ESR were analyzed in a stepwise logistic regression model. The results showed that serum LDH (odds ratio of 1.01, 95\% CI 1.00-1.01, $P<.001$ ) and ESR (odds ratio of $1.02,95 \% \mathrm{CI} 1.00-1.03, P<.001$ ) on admission were significant risk factors for refractory M. pneumoniae pneumonia (Table 2). In all covariates, we found that $\mathrm{LDH}$ and $\mathrm{HBDH}$ had a positive correlation, and the Pearson correlation was 0.822 ( $P=.01)$ (Fig. 2).

\section{ROC Curve Analysis}

The area under the curve for LDH was 0.718 (95\% CI $0.678-0.758$ ) as determined by ROC curve analysis (Fig. $3)$. The optimal cutoff of $\mathrm{LDH}$ for predicting refractory M. pneumoniae pneumonia was 379 IU/L, with a sensitivity of $48 \%$, specificity of $85.8 \%$, positive predictive value of $74.2 \%$, and negative predictive value of $65.9 \%$ (Table $3)$. The area under the curve for ESR was $0.683(95 \% \mathrm{CI}$ 0.626-0.740), and that for $\mathrm{HBDH}$ was 0.691 (95\% CI $0.650-0.733$ ) (Fig. 3 and Table 3).

\section{Discussion}

The incidence of refractory $M$. pneumoniae pneumonia has recently increased, ${ }^{4,16,17}$ and it is associated with macrolide-resistant strains. ${ }^{18-21}$ Cell-mediated immunity

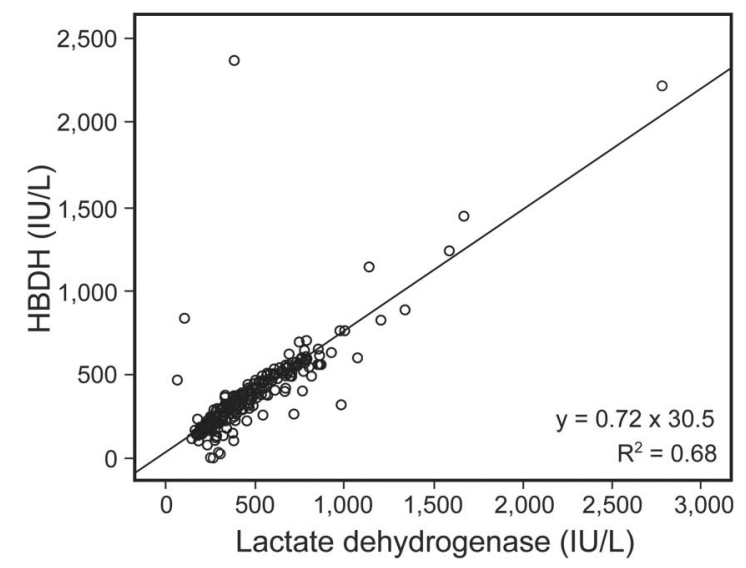

Fig. 2. Correlation between lactate dehydrogenase and $\alpha$-hydroxybutyrate dehydrogenase (HBDH).

of the host rather than direct microbial damage plays an important role in the progression of this illness. ${ }^{22,23}$ Clinically and experimentally, steroids have a positive effect on refractory $M$. pneumoniae pneumonia. ${ }^{2,4,16,24}$ However, steroids are usually initiated after the condition deteriorates. Thus, those with unrecoverable damage due to refractory $M$. pneumoniae pneumonia cannot rehabilitate completely. ${ }^{25-28}$ Although there is no case-control study on the benefit of steroids in refractory $M$. pneumoniae pneumonia, it is believed that early steroid therapy might prevent disease progression and reduce disease morbidity without adverse reactions. ${ }^{29,30}$ However, it is impossible to initiate steroids early for all $M$. pneumoniae infections because most of them are self-limited, and there is no evidence to support the use of systemic steroids in community-acquired pneumonia due to other pathogens. ${ }^{31}$ In addition, an epidemiological investigation showed that there is no apparent risk factor for refractory $M$. pneumoniae pneumonia ${ }^{32}$; therefore, predicting this illness at an early stage may improve the prognosis of M. pneumoniae infection, as this might help identify subjects for trials of early steroid administration.

Logistic regression showed that serum LDH and ESR were the main risk factors for refractory M. pneumoniae pneumonia after age difference was adjusted. Studies showed that LDH was associated with many pulmonary 

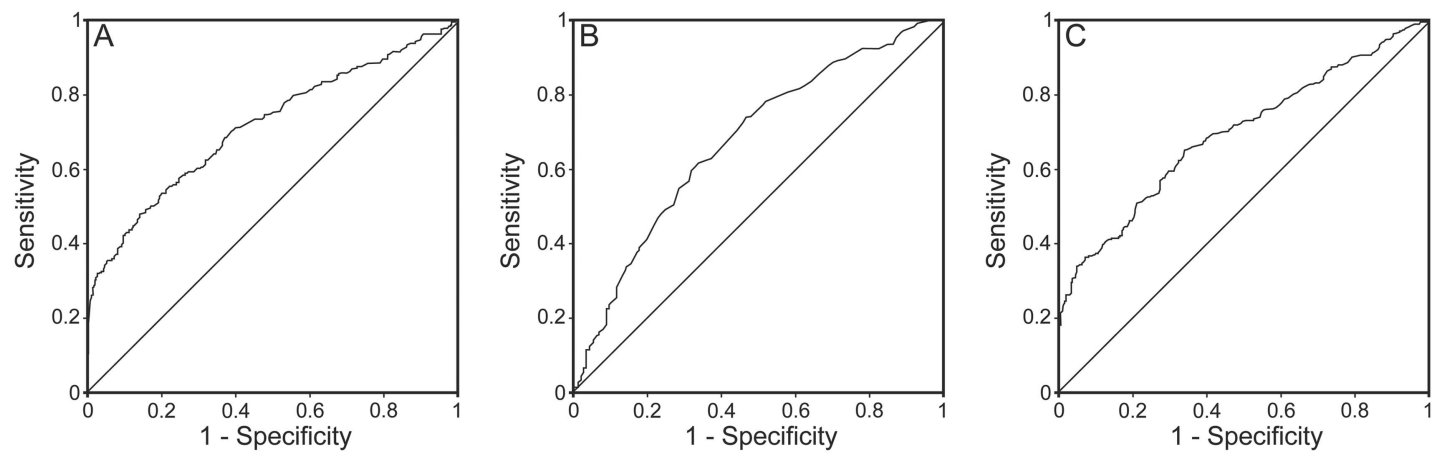

Fig. 3. Receiver operator characteristic curves for predicting refractory M. pneumoniae pneumonia. A: Lactate dehydrogenase. B: Erythrocyte sedimentation rate. C: $\alpha$-Hydroxybutyrate dehydrogenase.

Table 3. Predictive Value of LDH, ESR, and HBDH

\begin{tabular}{lcccccccc}
\hline \hline Parameter & $\begin{array}{c}\text { Area Under } \\
\text { the Curve }\end{array}$ & $\begin{array}{c}\text { Cutoff } \\
(\mathrm{IU} / \mathrm{L})\end{array}$ & $\begin{array}{c}\text { Sensitivity } \\
(\%)\end{array}$ & $\begin{array}{c}\text { Specificity } \\
(\%)\end{array}$ & $\begin{array}{c}\text { Positive } \\
\text { LR }(\%)\end{array}$ & $\begin{array}{c}\text { Negative } \\
\text { LR }(\%)\end{array}$ & $\begin{array}{c}\text { Positive Predictive } \\
\text { Value }(\%)\end{array}$ & $\begin{array}{c}\text { Negative Predictive } \\
\text { Value }(\%)\end{array}$ \\
\hline LDH & 0.718 & 379 & 48 & 85.8 & 3.37 & 0.61 & 74.2 & 65.9 \\
ESR & 0.683 & 32.5 & 62 & 66.4 & 1.84 & 0.57 & 61.2 & 67.1 \\
HBDH & 0.691 & 259.5 & 65 & 66.1 & 1.90 & 0.54 & 61.9 & 68.5
\end{tabular}

Receiver operating characteristic curve analysis was performed with suitable parameters to create cutoffs to determine the predictive value of each parameter with regard to refractory M. pneumoniae pneumonia.

$\mathrm{LDH}=$ lactate dehydrogenase

$\mathrm{ESR}=$ erythrocyte sedimentation rate

$\mathrm{HBDH}=\alpha$-hydroxybutyrate dehydrogenase

$\mathrm{LR}=$ likelihood ratio

diseases, such as obstructive diseases, microbial pulmonary diseases, and interstitial lung diseases. ${ }^{33,34}$ Several studies $^{2,4,13}$ also found that serum LDH was elevated in refractory M. pneumoniae pneumonia; however, there was no evidence showing that this elevation was associated with host immune response or virulence of $M$. pneumoniae. In this study, we found that the area under the curve for LDH was 0.718 in ROC curve analysis, indicating fair discriminative power for predicting refractory M. pneumoniae pneumonia. The optimal cutoff for LDH was $379 \mathrm{IU} / \mathrm{L}$, with a sensitivity of $48 \%$ and specificity of $85.8 \%$. This indicates its clinical utility in identifying patients at high risk for refractory $M$. pneumoniae pneumonia: namely, patients with usual $M$. pneumoniae pneumonia will not be misdiagnosed with refractory pneumonia and started on steroid therapy. Another study showed that children with M. pneumoniae infection who were possible candidates for steroid therapy usually had significantly elevated levels of LDH (> $480 \mathrm{IU} / \mathrm{L}){ }^{35}$ Inamura et $\mathrm{al}^{13}$ performed ROC curve analysis and showed that the cutoff of LDH for refractory M. pneumoniae pneumonia was $410 \mathrm{IU} / \mathrm{L}$. The cutoffs were greater than in our study. The main reason may be that their results were obtained from a small case series, and they had more serious illnesses. In this study, at the cutoff point, the positive predictive value of LDH was
$74.2 \%$. As positive predictive value is also sensitive to disease prevalence, which may be different in different clinical settings, the LDH test may have a lower positive predictive value in populations with a lower prevalence of refractory $M$. pneumoniae pneumonia.

The serum HBDH test measures mainly the LDH-1 and LDH-2 isoforms, reflecting serum LDH activity. ${ }^{36}$ Few reports are available on the serum activity of $\mathrm{HBDH}$ in pulmonary disease. In this study, we found that $\mathrm{HBDH}$ was associated with refractory $M$. pneumoniae pneumonia and could be used as a potential predictive marker for this illness. However, ROC curve analysis in this study showed that it was not an ideal biomarker for predicting refractory M. pneumoniae pneumonia. In addition, we found that $\mathrm{HBDH}$ was positively correlated with LDH in M. pneumoniae infections, which may be the reason why HBDH was not a risk factor in the logistic regression analysis. To our knowledge, this is the first report on the association between HBDH and refractory M. pneumoniae pneumonia.

There are limitations to our study. First, we divided our subjects into 2 groups based on the definition of refractory M. pneumoniae pneumonia, ${ }^{2,16}$ and this definition was broad. Therefore, spectrum bias was introduced into our results. Moreover, this bias may be the main reason why the ROC curve analysis of LDH showed 


\section{Lactate Dehydrogenase as a Biomarker for M. pneumoniae Pneumonia}

just a fair discriminative power for refractory $M$. pneumoniae pneumonia. However, the children in the refractory pneumonia group remained at the highest risk for severe or refractory illness. Thus, our findings could still be applied to a very common clinical scenario for screening those refractory cases.

\section{Conclusions}

In summary, serum LDH can be used as a biomarker for predicting refractory $M$. pneumoniae pneumonia and determining candidates who may benefit from corticosteroid therapy during the early stages of hospitalization. Furthermore, more research is needed to identify the potential utility of early steroid administration, which could be performed based on the use of $\mathrm{LDH}$ as a biomarker to initiate steroid treatment.

\section{ACKNOWLEDGMENTS}

We gratefully acknowledge the statistical help given by Mr Peng Shi (Department of Information Management, Children's Hospital of Fudan University, and Center of Evidence-based Medicine, Fudan University, Shanghai, China). We also acknowledge LetPub (www.letpub.com) for linguistic assistance during the preparation of this manuscript.

\section{REFERENCES}

1. Lee KY. Pediatric respiratory infections by Mycoplasma pneumoniae. Expert Rev Anti Infect Ther 2008;6(4):509-521.

2. Lee KY, Lee HS, Hong JH, Lee MH, Lee JS, Burgner D, Lee BC. Role of prednisolone treatment in severe Mycoplasma pneumoniae pneumonia in children. Pediatr Pulmonol 2006;41(3):263-268.

3. Miyashita N, Obase Y, Ouchi K, Kawasaki K, Kawai Y, Kobashi Y, Oka M. Clinical features of severe Mycoplasma pneumoniae pneumonia in adults admitted to an intensive care unit. J Med Microbiol 2007;56(Pt 12):1625-1629.

4. Lu A, Wang L, Zhang X, Zhang M. Combined treatment for child refractory Mycoplasma pneumoniae pneumonia with ciprofloxacin and glucocorticoid. Pediatr Pulmonol 2011;46(11):1093-1097.

5. Zhou Y, Zhang Y, Sheng Y, Zhang L, Shen Z, Chen Z. More complications occur in macrolide-resistant than in macrolide-sensitive Mycoplasma pneumoniae pneumonia. Antimicrob Agents Chemother 2014;58(2):1034-1038.

6. Wanat KA, Castelo-Soccio L, Rubin AI, Treat JR, Shah KN. Recurrent Stevens-Johnson syndrome secondary to Mycoplasma pneumoniae infection. Cutis 2014;93(4):E7-E8.

7. Vujic I, Shroff A, Grzelka M, Posch C, Monshi B, Sanlorenzo M, et al. Mycoplasma pneumoniae-associated mucositis-case report and systematic review of literature. J Eur Acad Dermatol Venereol 2015;29(3):595-598.

8. Vieira-Baptista P, Machado L, Costa AR, Beires J, Martinez-deOliveira J. Mycoplasma pneumoniae: a rare cause of vulvar ulcers or an undiagnosed one? J Low Genit Tract Dis 2013;17(3):330-334.

9. Vargas-Hitos JA, Manzano-Gamero MV, Jiménez-Alonso J. Erythema multiforme associated with Mycoplasma pneumoniae. Infection 2014;42(4):797-798.

10. Kim GH, Seo WH, Je BK, Eun SH. Mycoplasma pneumoniae associated stroke in a 3-year-old girl. Korean J Pediatr 2013;56(9):411415 .
11. Hanzawa F, Fuchigami T, Ishii W, Nakajima S, Kawamura Y, Endo A, et al. A 3-year-old boy with Guillain-Barre syndrome and encephalitis associated with Mycoplasma pneumoniae infection. J Infect Chemother 2014;20(2):134-138.

12. You SY, Jwa HJ, Yang EA, Kil HR, Lee JH. Effects of methylprednisolone pulse therapy on refractory Mycoplasma pneumoniae pneumonia in children. Allergy Asthma Immunol Res 2014;6(1): 22-26.

13. Inamura N, Miyashita N, Hasegawa S, Kato A, Fukuda Y, Saitoh A, et al. Management of refractory Mycoplasma pneumoniae pneumonia: utility of measuring serum lactate dehydrogenase level. J Infect Chemother 2014;20(4):270-273.

14. Seo YH, Kim JS, Seo SC, Seo WH, Yoo Y, Song DJ, Choung JT. Predictive value of $\mathrm{C}$-reactive protein in response to macrolides in children with macrolide-resistant Mycoplasma pneumoniae pneumonia. Korean J Pediatr 2014;57(4):186-192.

15. Subspecialty Group of Respiratory Diseases, The Society of Pediatrics, Chinese Medical Association, Editorial Board, Chinese Journal of Pediatrics. [Guidelines for management of community acquired pneumonia in children (the revised edition of 2013) (I)]. Zhonghua Er Ke Za Zhi 2013;51(10):745-752. Article in Chinese.

16. Tamura A, Matsubara K, Tanaka T, Nigami H, Yura K, Fukaya T. Methylprednisolone pulse therapy for refractory Mycoplasma pneumoniae pneumonia in children. J Infect 2008;57(3):223-228.

17. Daxboeck F, Eisl B, Burghuber C, Memarsadeghi M, Assadian O, Stanek G. Fatal Mycoplasma pneumoniae pneumonia in a previously healthy 18-year-old girl. Wien Klin Wochenschr 2007;119(11-12): 379-384.

18. Liu Y, Ye X, Zhang H, Xu X, Li W, Zhu D, Wang M. Antimicrobial susceptibility of Mycoplasma pneumoniae isolates and molecular analysis of macrolide-resistant strains from Shanghai, China. Antimicrob Agents Chemother 2009;53(5):2160-2162.

19. Miyashita N, Akaike H, Teranishi H, Ouchi K, Okimoto N. Macrolide-resistant Mycoplasma pneumoniae pneumonia in adolescents and adults: clinical findings, drug susceptibility, and therapeutic efficacy. Antimicrob Agents Chemother 2013;57(10):5181-5185.

20. Kawai Y, Miyashita N, Kubo M, Akaike H, Kato A, Nishizawa Y, et al. Nationwide surveillance of macrolide-resistant Mycoplasma pneumoniae infection in pediatric patients. Antimicrob Agents Chemother 2013;57(8):4046-4049.

21. Zhao H, Li S, Cao L, Yuan Y, Xue G, Feng Y, et al. Surveillance of Mycoplasma pneumoniae infection among children in Beijing from 2007 to 2012. Chin Med J 2014;127(7):1244-1248.

22. Waites KB, Talkington DF. Mycoplasma pneumoniae and its role as a human pathogen. Clin Microbiol Rev 2004;17(4):697-728.

23. Yang J, Hooper WC, Phillips DJ, Talkington DF. Cytokines in $M y$ coplasma pneumoniae infections. Cytokine Growth Factor Rev 2004; 15(2-3):157-168.

24. Tagliabue C, Salvatore CM, Techasaensiri C, Mejias A, Torres JP, Katz K, et al. The impact of steroids given with macrolide therapy on experimental Mycoplasma pneumoniae respiratory infection. J Infect Dis $2008 ; 198(8): 1180-1188$.

25. Tran H, Allworth A, Bennett C. A case of Mycoplasma pneumoniaeassociated encephalomyelitis in a 16-year-old female presenting to an adult teaching hospital. Clin Med Insights Case Rep 2013;6:209211.

26. Santiago-Burruchaga M, Zalacain-Jorge R, Alvarez-Martinez J, Arguinzoniz-Marzana JM, Pocheville-Guruzeta I, Vazquez-Ronco MA, et al. Hereditary pulmonary alveolar proteinosis. Could it be triggered by Mycoplasma pneumoniae pneumonia? Respir Med 2013; 107(1):134-138.

27. Lee CY, Huang YY, Huang FL, Liu FC, Chen PY. Mycoplasma pneumoniae-associated cerebral infarction in a child. J Trop Pediatr 2009;55(4):272-275. 


\section{Lactate Dehydrogenase as a Biomarker for M. pneumoniae Pneumonia}

28. Lee M, Joo IS, Lee SJ, Hong JM, Lee JS. Multiple cerebral arterial occlusions related to Mycoplasma pneumoniae infection. Neurol Sci 2013;34(4):565-568.

29. Miyashita N, Kawai Y, Inamura N, Tanaka T, Akaike H, Teranishi $\mathrm{H}$, et al. Setting a standard for the initiation of steroid therapy in refractory or severe Mycoplasma pneumoniae pneumonia in adolescents and adults. J Infect Chemother 2015;21(3):153-160.

30. Youn YS, Lee SC, Rhim JW, Shin MS, Kang JH, Lee KY. Early additional immune-modulators for Mycoplasma pneumoniae pneumonia in children: an observation study. Infect Chemother 2014;46(4):239-247.

31. Shafiq M, Mansoor MS, Khan AA, Sohail MR, Murad MH. Adjuvant steroid therapy in community-acquired pneumonia: a systematic review and meta-analysis. J Hosp Med 2013;8(2):68-75.

32. Izumikawa K, Takazono T, Kosai K, Morinaga Y, Nakamura S, Kurihara S, et al. Clinical features, risk factors and treatment of fulminant Mycoplasma pneumoniae pneumonia: a review of the Japanese literature. J Infect Chemother 2014;20(3):181-185.
33. Drent M, Cobben NA, Henderson RF, Wouters EF, van DieijenVisser M. Usefulness of lactate dehydrogenase and its isoenzymes as indicators of lung damage or inflammation. Eur Respir J 1996;9(8):1736-1742.

34. Nakajima M, Kawahara Y, Yoshida K, Miyashita N, Niki Y, Matsushima T. Serum KL-6 as a possible marker for amiodaroneinduced pulmonary toxicity. Intern Med 2000;39(12):10971100.

35. Oishi T, Narita M, Matsui K, Shirai T, Matsuo M, Negishi J, et al. Clinical implications of interleukin-18 levels in pediatric patients with Mycoplasma pneumoniae pneumonia. J Infect Chemother 2011; 17(6):803-806.

36. Dissmann R, Linderer T, Schröder R. Estimation of enzymatic infarct size: direct comparison of the marker enzymes creatine kinase and $\alpha$-hydroxybutyrate dehydrogenase. Am Heart J 1998; 135(1):1-9. 